\title{
MATERIA E FORMA DEI MITI. \\ SU MIMESI PLATONICA E SIMBOLOGIA OMERICA
}

\author{
Anna Motta ${ }^{1}$
}

\begin{abstract}
Sommario
L'interferenza di mito, religione e pensiero razionale caratterizza quella filosofia che, nella tarda antichità, si configura come una mistagogia e al contempo come una scienza teologica. Obiettivo di questo paper è quello di offrire un quadro sulle discussioni neoplatoniche attorno al mito e al rapporto tra Platone e i poeti-teologi, che, banditi dalla kallipolis, sono riabilitati nei testi della tarda antichità. La riflessione sul linguaggio della scienza e quella sul linguaggio del simbolo sono certamente alcune delle chiavi di lettura attraverso le quali è possibile comprendere l'operazione di recupero del mito omerico e l'attribuzione di caratteri di scientificità divina alla mimesis platonica.
\end{abstract}

Parole chiave: mistagogia, mito, linguaggio, decoro, oscenità.

\begin{abstract}
The connection among myth, religion and rational thought characterizes that philosophy which, in Late Antiquity, seems to be a mystagogy and at the same time a theological science. The aim of this paper is to present critically some of the discussions on myth and on the relationship between Plato and the poets-theologians, who, banished from the kallipolis, are rehabilitated in the Late Antique texts. The analysis of the language of the science and that on the language of the symbol are certainly some reading keys through which it is possible to grasp the activity of rehabilitation of the Homeric myth and the divine scientificity of the Platonic mimesis.
\end{abstract}

Keywords: mystagogy, myth, language, propriety, obscenity.

\footnotetext{
${ }^{1}$ Anna Motta has obtained her Degrees in Classical Studies and Ancient Philology at the University of Naples "Federico II" and her PhD title with a thesis concerning Neoplatonism and Late Antique philosophy at the University of Salerno. She has also taken part in Internatonal conferences and she has written articles especially about Neoplatonism and a book (Prolegomeni alla Filosofia di Platone, Armando editore, Roma 2014), in which she shows how different philosophies and religious traditions go together and become harmonized under the name of Plato.
} 


\section{PREMESSA}

Sin dai primi secoli dell'impero i Platonici si sforzano di riconciliare diverse tradizioni teologiche riuscendo, nella tarda antichità, a riempire la presunta distanza tra filosofia e mitologia, tra $\operatorname{logos}$ e $m y$ thos $^{2}$, tra pensiero razionale e misticismo religioso. In realtà, Giorgio Colli ha ben evidenziato come, già dalle origini più lontane e non solo in epoca imperiale e poi col neoplatonismo, la compresenza di un aspetto razionale e di uno mistico possano manifestarsi in uno stesso pensatore o in una stessa corrente (COLLI 1975). Seguendo questa linea interpretativa la tradizione poetico-religiosa rappresenta non solo l'inizio, ma la continua fonte cui attinge l'intera sapientia filosofica greca, la quale non è semplicemente l'esito né di una brusca né di una progressiva emancipazione dal mito ${ }^{3}$. Francis MacDonald Cornford è stato tra i primi a combattere "la teoria del miracolo greco", teoria che presenta la fisica ionica come rivelazione improvvisa e incondizionata della ragione $^{4}$. Gli studi di Jean-Pierre Vernant hanno poi dimostrato che il mito non è mai privo di logos, perché esso entra sempre in relazione, benché in maniera controversa, col pensiero razionale e non scompare con l'elaborazione delle prime scienze. Infatti la contrapposizione tra mythos e logos - scrive Lo Schiavo - «passa storicamente, attraverso la reciproca interferenza dei due differenti piani: la mitologia orientando nella sua direzione la 'fisica' degli Ionici, il pensiero 'logico' tentando di "conformare ai propri criteri i racconti mitici" (LO SCHIAVO 1983: 241)». L'interferenza di mito e pensiero razionale non caratterizza soltanto il fondamento naturalistico e razionale comune alla religione olimpica e a quella parte della filosofia non influenzata dalle correnti mistiche. Anzi, il misticismo della tarda antichità conserva in sé una matrice razionale di natura esegetica - fondata sulla interpretazione dei dialoghi di Platone ${ }^{5}$ che non chiudono l'uomo nei limiti dell'umano - e una irrazionale legata a riti purificatori.

\footnotetext{
${ }^{2}$ Sulle relazioni tra mythos e logos nell'antichità e nella tarda antichità vastissima è la bibliografia: cfr., p.e., BUFFIÈRE 1956; PÉPIN 1958; DETIENNE 1983; RISPOLI 1988; CERRI 1991; SAFFREY 1992; BRISSON 1996; KUISMA 1996; BUXTON 1999; CALAME 1999; FERRARI 2006; VERNANT 2007.

${ }^{3}$ Per una ricostruzione storica del legame tra mito e filosofia da Platone al Rinascimento cfr. BRISSON 2004.

${ }^{4}$ CORNFORD 1952.

${ }^{5} \mathrm{Cfr}$. Pl. Tht. 176b 1. Il tema della homiosis theo( $\left.i\right)$ è costante, seppur venga usato in maniera diversificata, nella speculazione medio e neo-platonica: cfr. LAVECCHIA 2006.
} 


\title{
FILOSOFIA E MISTAGOGIA
}

La filosofia antica sin dai Presocratici tende a definire se stessa come una "iniziazione”, iniziazione che si inserisce nel solco degli antichi misteri. Nel Fedone l'iniziato è chi ha filosofato anche se non tutti i filosofi raggiungono il supremo grado iniziatico come i Bacchi, cioè come coloro che nei riti dionisiaci sono completamente posseduti dal dio:

\begin{abstract}
$\mathrm{E}$ anche quei tali che istituirono i misteri non pare fossero gente stolta; e in verità già da tempo, per via degli enigmi, ci hanno fatto intendere che chi giunga nell'Ade senza aver partecipato ai Misteri né compiuta la sua iniziazione, costui giacerà nel fango, e invece chi vi giunga in tutto purificato e iniziato, egli vivrà in compagnia degli dèi. Ché veramente, come dicono gli iniziatori di questi Misteri, «molti sono che portano le fèrule, ma Bacchi pochi». E questi sono, a mio credere, non altri che coloro i quali hanno esercitato filosofia nel vero senso della parola (Pl. Phd. 69c 3-d 3 ; trad. CENTRONE 1991) ${ }^{6}$.
\end{abstract}

A distanza di secoli i Neoplatonici Plotino, Proclo e Marino stabiliscono, a chiare lettere, una relazione tra Platone e gli antichi testi religiosi:

D'altra parte questi discorsi non sono nuovi né si fanno soltanto ora, ma furono fatti già dagli antichi, benché non in modo esplicito; quel che ora diciamo è un'intepretazione di questi discorsi, un'interpretazione che, appoggiandosi alla testimonianza degli scritti dello stesso Platone, conferma l'antichità di tali opinioni (Plot. V 1 [10], 8.10-14; trad. GUIDELLI 1997) ${ }^{7}$.

Infatti tutta quanta la teologia presso i Greci è proveniente dalla dottrina mistica Orfica, dato che Pitagora per primo fu istruito da Aglaofemo nei riti sacri concernenti gli dèi, mentre Platone per secondo ricevette la perfetta scienza degli dèi dagli scritti Pitagorici e Orfici (Procl. Theol. Plat. I 5, 25.26-26.4; trad. ABBATE 2005).

Dai testi dei Neoplatonici è possibile anche individuare tappe didattiche del cursus studiorum il raggiungimento delle quali ci aiuta a distinguere aspiranti Platonici e iniziati:

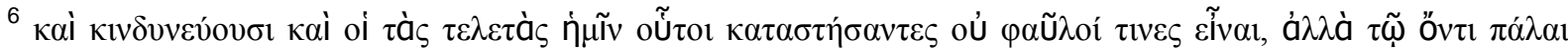

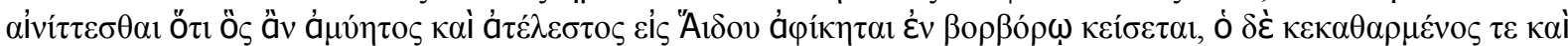

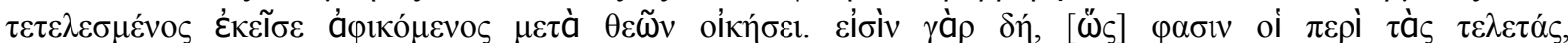

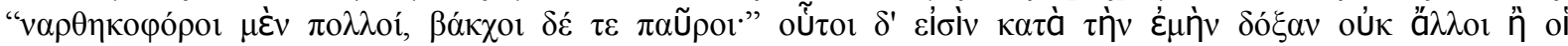
$\pi \varepsilon \varphi \imath \lambda о \sigma o \varphi \eta \kappa o ́ \tau \varepsilon \zeta ~ o ́ \rho \theta \tilde{\omega} \varsigma$.

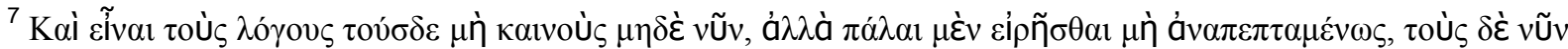

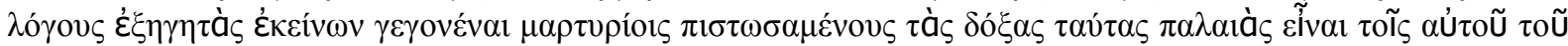
$\Pi \lambda \alpha \dot{\tau} \tau \omega v \sigma \varsigma \gamma \rho \alpha ́ \alpha \mu \alpha \sigma \mathrm{v}$. 
In meno di due anni [Proclo] lesse insieme con lui [scil. Siriano] tutti i trattati di Aristotele, sulla logica, sull'etica, sulla politica, sulla fisica e sulla teologia che è scienza al di sopra di quelle. Dopo averlo guidato adeguatamente fra di esse, come attraverso certi riti iniziatori e piccoli misteri, Siriano lo avviò alla mistagogia di Platone, procedendo con ordine e, come dice l'oracolo, non oltrepassando i limiti; $e$ gli fece contemplare con gli occhi limpidi dell'anima e con lo sguardo puro della ragione $i$ misteri veramente divini che quel filosofo celebra (Marin. 13; trad. MASULLO 1985, lievemente modificata) ${ }^{8}$.

I tre passaggi sono significativi per poter affermare che se l'inizio della teologia greca è la mistagogia orfica della quale Pitagora e Platone sono seguaci, allora tutta la filosofia, che è stata in relazione e si è sviluppata da tale saggezza orfica, può definirsi correttamente 'mistagogia', termine che è esplicitamente riferito alla dottrina platonica, ai «grandi misteri», per la prima volta qui da Marino ${ }^{10}$. Proclo, maestro di Marino, rimarca il legame tra gli Orfici e Pitagora poiché è sua convinzione che i princìpi di Pitagora siano gli insegnamenti ricevuti dagli Orfici nel corso della loro iniziazione ${ }^{11}$. Ed è sempre Proclo a sottolinearne anche il carattere esoterico ${ }^{12}$. Attraverso l'immagine di un giovane Platone che sulle orme dei Pitagorici apprende l'arte ieratica recandosi in Egitto, proprio perché i Pitagorici - che poi specifica hanno imparato in Persia la saggezza dei Magi ${ }^{13}$ - provenivano da questa terra ${ }^{14}$, l'anonimo autore dei Prolegomeni a Platone recepisce e accetta di divulgare, calandole in un preciso contesto esegetico, testimonianze dossografiche e spunti tratti dai dialoghi che paragonano le iniziazioni e i dogmi pitagorici a quelli di Platone, dei Magi e degli Egiziani.

L'identificazione della filosofia con la teologia trasmessa dal misterioso Aglaofemo non è, però, una novità procliana, bensì è la ripresa ateniese del programma annunciato dalla

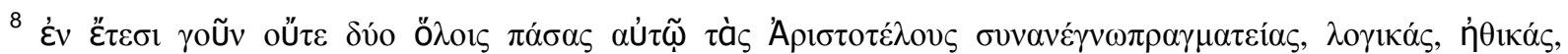

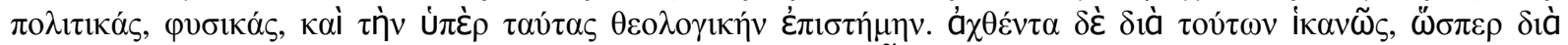

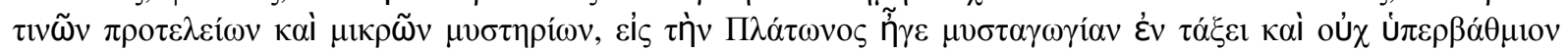

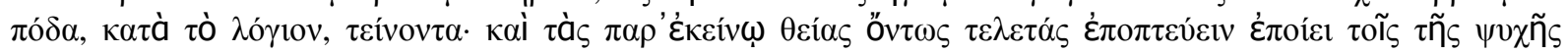

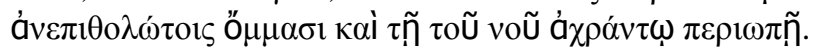

${ }^{9}$ Cfr. Procl. Theol. Plat. I 6, 25.24-26.4.

10 Sulla funzione della filosofia in Plotino cfr. TROUILLARD 1982: 27-32. Per l'interpretazione della mistagogia procliana e dei simboli mitici attraverso Marino cfr. ancora TROUILLARD 1982: 33-51, in part. 4651.

${ }^{11}$ Cfr. Procl. in Ti. III 169, 8-20.

${ }^{12}$ Anche Platone (R. 2, 364e-365 a) fa allusione a cerimonie celebrate da persone che si richiamano a Orfeo.

${ }^{13}$ Cfr. Anon. Proll. 6.24-25. Diogene Laerzio riferisce che Aristotele «nel primo libro dell'opera Della filosofia afferma che i Magi erano ancora più antichi degli Egizi».

${ }^{14}$ Cfr. Anon. Proll. 4.9-12 e Olymp. in Alc. 2.134-135. 
Vita Pitagorica, biografia in cui Giamblico collega - secondo Brisson, per la prima volta ${ }^{15}$ Pitagora all'orfismo:

\begin{abstract}
Non c'è dubbio che Pitagora prese spunto da Orfeo nello scrivere il discorso Sugli dèi che proprio per questo denonimò «sacro», perché rappresentava per così dire il fior fiore tratto dai più arcani recessi della dottrina di Orfeo; un'opera, questa, che realmente è di Pitagora [...]. Ora il Discorso sacro in questione (ovvero l'opera Sugli dèi: sono noti entrambi i titoli) mostra chiaramente chi trasmise a Pitagora quanto in esso si dice circa gli dèi. Il testo suona così: «Questo è il discorso sugli dèi di Pitagora figlio di Mnemarco, che io, Pitagora, appresi quando fui iniziato ai misteri nella tracia di Libetro. Aglaofamo, sacerdote responsabile delle iniziazioni, mi rivelò che Orfeo, figlio di Calliope, il quale era stato istruito da sua madre sul monte Pangeo, aveva detto: l'essenza eterna del numero è il principio provvidenzialissimo dell'universo cielo, della terra e della natura intermedia tra l'uno e l'altro, ed è anche la radice che consente ai mortali, agli dèi, ai dèmoni di continuare a esistere» (Iamb. VP 146; trad. GIANGIULO 1991) ${ }^{16}$.
\end{abstract}

La Vita Pitagorica è quindi il luogo testuale nel quale si può rinvenire una chiara relazione tra Orfeo, Pitagora e Platone, luogo che dà inizio a esegesi armoniche del pensiero dei tre "teologi". Tale relazione, assieme a quella con altre tradizioni teologiche, è resa esplicita da Siriano in opere purtroppo perdute, ma i cui titoli - la Teologia di Orfeo e l'Accordo tra Orfeo, Pitagora, Platone con gli Oracoli Caldaici - sono oltremodo indicativi ${ }^{17}$. L'interesse per il pensiero mitico e religioso non viene tradito dal suo successore Proclo, il quale, benché non scriva un commento completo dei Poemi di Orfeo ${ }^{18}$ - come aveva fatto per gli Oracoli - continua a mostrarsi fortemente interessato e affascinato da ogni corrente teologica. Proclo - si legge nella sua biografia - studia presso Siriano e sceglie di leggere, su suggerimento del maestro, gli Oracoli Caldaici e i commenti di Porfirio e Giamblico a questi testi sacri. È con tale bagaglio che il diadoco ateniese fa esegesi e

\footnotetext{
${ }^{15}$ Cfr. BRISSON, 2000, pp. 237-253.

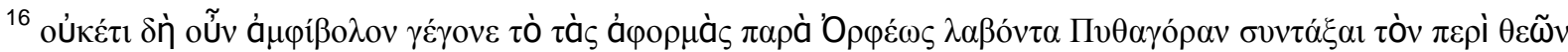

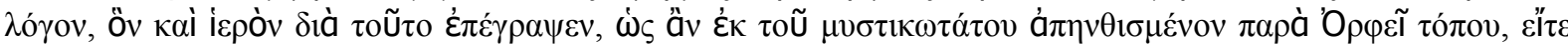

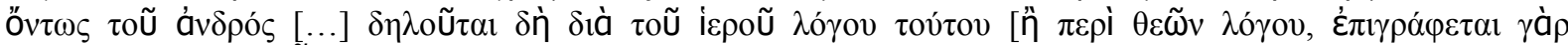

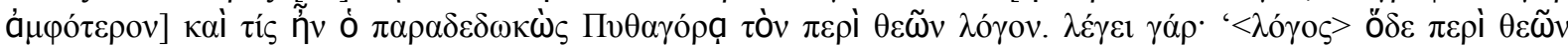

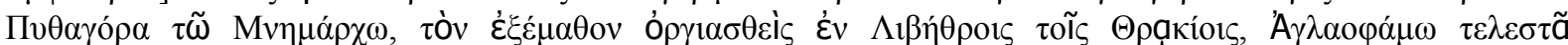

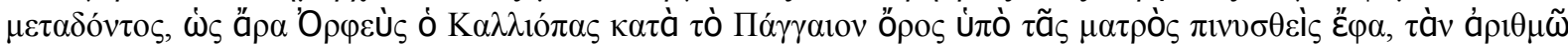

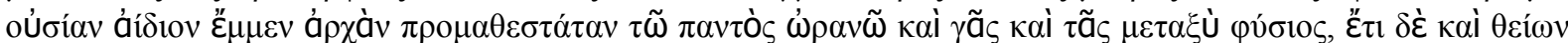

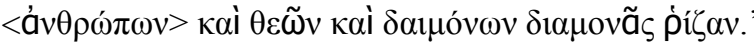

${ }^{17}$ Su Siriano cfr. MANOLEA 2004.

${ }^{18}$ Cfr. Marin. 27.
} 
aggiunge al lavoro dei filosofi che lo avevano preceduto le argomentazioni della dottrina caldaica $^{19}$.

L'interesse per le varie teologie, che si armonizzano alla mistagogia platonica, evidenzia un aspetto molto interessante della speculazione della tarda antichità: la filosofia, dono della divinità a un gruppo di iniziati, è una verità rivelata trasmessa in Grecia da figure di sapienti quali Orfeo, Pitagora, Platone e i teurgi, in luoghi distanti geograficamente e culturalmente, come l'Egitto e la Grecia, e tra popoli diversi per tradizioni, costumi e lingua. Divina è la filosofia, in quanto Platone che l'ha condotta a perfezione è divino: per tal motivo essa deve essere insegnata e divulgata secondo precise modalità.

Tuttavia va detto che anche quando la filosofia non intende esplicitamente se stessa come iniziazione misterica, definisce comunque - spiega Pierre Hadot - se stessa come arte, come esercizio spirituale, il cui scopo è la cura dell'anima (HADOT 1987). Gli studi dell'intellettuale francese dimostrano che il modo migliore di intendere la filosofia antica è quello di considerarla come un esercizio spirituale e come una medicina dell'anima. La sua teoria è paradossalmente più convincente se applicata alle scuole ellenistiche, quando cioè i filosofi sembrano prediligere alla disciplina religiosa e spirituale un rigore puramente etico, e funziona perfettamente se applicata alla tarda antichità, quando la riflessione platonica si apre a influenze orfico-pitagoriche e caldaiche e definisce se stessa una teologia.

\begin{abstract}
A tali virtù [Proclo] conformò massimamente la propria vita e fece in ogni occasione quanto potesse consentire all'anima la separazione dal corpo: notte e giorno ricorreva a scongiuri, aspersioni e altre purificazioni, di natura ora orfica ora caldaica [...]. A queste pratiche abitudinarie non solo attese con perseveranza nel fiore della vita, ma si dedicò anche quando ormai si approssimava la fine, osservandole senza interruzione come una legge (Marin. 18; trad. MASULLO $1985)^{20}$.
\end{abstract}

L'interesse di molti Platonici dell'antichità tardiva per la teurgia ${ }^{21}$, intesa come pratica di cura dell'anima e metodo di purificazione e accesso al divino e, quindi, la

\footnotetext{
${ }^{19}$ Cfr. Marin. 26.

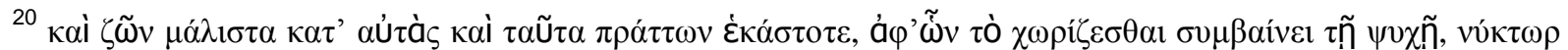

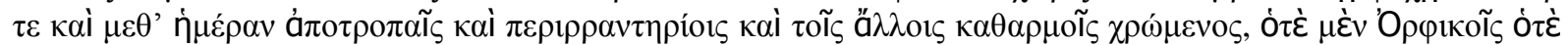

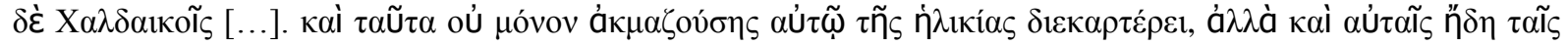

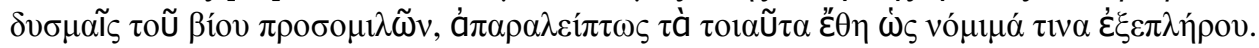

${ }^{21}$ Cfr. Procl. in R. I 81.11-21. La dipendenza di Proclo dagli Oracoli Caldaici, oltre a essere da lui stesso ammessa (in R. I 11.1-4), è attestata da Marino (28), il quale dice che egli, come Giuliano il Caldeo, riteneva di appartenere alla catena ermetica. Cfr. SAFFREY 1981 211-225.
} 
sovrapposizione di filosofia e teurgia, è cosa nota, ma è degno di essere approfondito anche come a tale interesse possa legarsi il tentativo esegetico di recuperare i miti omerici nel loro valore simbolico, nascosto ai più e accessibile agli iniziati ${ }^{22}$. I Neoplatonici devono, infatti, superare le "apparenti" contraddizioni presenti nei dialoghi del Maestro e spiegare, in primis, la critica platonica ai miti poetici, critica che sembra, senza mezzi termini, bandirli dalla kallipolis sia che essi abbiano un significato nascosto sia che non lo abbiano ${ }^{23}$. Tuttavia bandire Omero per i Neoplatonici vorrebbe dire mettere in discussione tutta la paideia antica e il progetto di scienza teologica che essi stanno costruendo ${ }^{24}$. In realtà, Platone non avrebbe potuto farlo, non avrebbe potuto rinnegare ciò che era parte del patrimonio culturale greco, in quanto ben cosciente - come mostrano gli anonimi Prolegomeni alla filosofia di Platone - di aver appreso egli stesso come esaltare l'ordine degli esseri proprio dai poeti-teologi ${ }^{25}$ : è infatti piuttosto una lettura degli studiosi moderni, ma non degli autori tardoantichi, quella che separa nettamente i campi del sapere e che distingue tra filosofia e teologia ${ }^{26}$.

Come mostrano gli studi di Lamberton, Brisson e Pichler, Proclo vede in Omero un poeta ispirato e un teologo e lo stesso Platone è presentato come poeta, filosofo e teologo ${ }^{27}$. Il rilievo di questo pensiero, che apprendiamo dagli scritti procliani, ma che probabilmente è precedente a lui e al suo stesso maestro Siriano, è evidente dal fatto che viene accolto anche nella scuola neoplatonica di Alessandria. Nei Prolegomeni si legge:

Di tutti questi filosofi Platone è stato considerato il migliore. Imparò in effetti dalla scuola poetica a esaltare l'ordine degli esseri, ma la superò per il fatto che i poeti parlano senza dimostrazione e, come egli stesso dice, «con bocca folle e ispirata» ${ }^{28}$,

\footnotetext{
22 Fondamentale, per dare inizio al dibattito, è stato lo studio di SHEPPARD 1980, seguito da BOUFFARTIGUE 1987; LAMBERTON 1992; KUISMA 1996; VAN DEN BERG 2001; MANOLEA 2004.

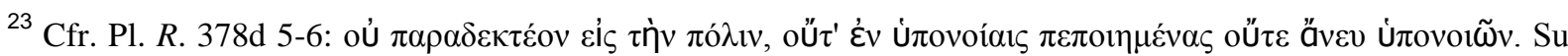
questo aspetto cfr. RAMELLI - LUCCHETTA 2004: 59; PICHLER 2006: 30, n. 55.

${ }^{24}$ Per la ricostruzione dei caratteri e della fisionomia della "scienza teologica" cfr. SAFFREY 1992: 35-50; DÖRRIE - BALTES - PIETSCH 2008.

${ }^{25}$ Cfr. Anon. Proll. 7.20

${ }^{26}$ Contro questa lettura moderna cfr. LO SCHIAVO, 1983 che discute di un Omero filosofo.

27 Cfr. LAMBERTON 1986: 22-31; BRISSON 1996: 122; PICHLER 2006: 67-68. Dall'antichità classica, attraverso Plutarco e i commentatori aristotelici, fino a Ermia, teologo e teologia hanno lo stesso significato che avevano avuto già a partire da Aristotele: il teologo è colui che parla di dio perché ispirato dal dio stesso e tali furono, secondo Aristotele, i più antichi poeti della Grecia (cfr. MORESCHINI 1996).
}

${ }^{28} \mathrm{Heracl}$. DK22B 92. Sull'uso neoplatonico di questo frammento cfr. MOTTA 2014: 104 n. 90 e la bibliografia ivi citata. 
lui invece interamente con dimostrazione (Anon. Proll. 7.19-23; trad. MOTTA $2014)^{29}$.

I filosofi cui si riferisce l'anonimo testo sono Orfeo, figlio di Calliope, una delle Muse, Omero, poeta cui le Muse tolgono la vista sensibile per donargli quella intelligibile ${ }^{30}$, Museo ed Esiodo che riceve l'investitura poetica dalle Muse d'Olimpo presso il monte Elicona $^{31}$. Il confronto di Platone con questi poeti è costruito probabilmente anche sulla base del passaggio procliano del Commento alla Repubblica in cui si legge che colui il quale è «ispirato dal dio e in preda al furore bacchico provocato dalle Muse ci insegna sulle cose divine e su quelle umane (Procl. in R. I 159, 1-3)» ${ }^{32}$.

Il testo procliano si riferisce chiaramente a Omero, poeta divinamente ispirato dalle Muse e la cui ispirazione è il prodotto della mania $^{33}$ della quale parla la pagina di Fedro 245a, pagina cui rimandano gli anonimi Prolegomeni citando il frammento eracliteo. Cosa ancora più interessante è il fatto che il poeta cieco, posseduto dalle Muse $^{34}$, abbia una relazione diretta con gli dèi: egli dice la verità per bocca della divinità ${ }^{35}$, quella stessa verità che Platone ha appresso e che, però, comunica attraverso miti filosofici. Platone è presentato nei Prolegomeni come il più filosofico dei poeti e il più ispirato dei filosofi: benché il suo proprio linguaggio sia dimostrativo ${ }^{36}$, la filosofia - che nella sua espressione più perfetta si identifica in quella di Platone - è in grado di servirsi di un linguaggio diverso a seconda dei differenti gradi di iniziazione raggiunti. Pertanto, se la verità è unica e sia Omero che Platone sono divini, perché vicini a essa, allora la differenza tra il massimo poeta e il più grande filosofo riguarda il linguaggio piuttosto che il contenuto ${ }^{37}$, l'aspetto esterno piuttosto che quello

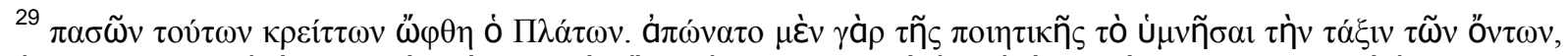

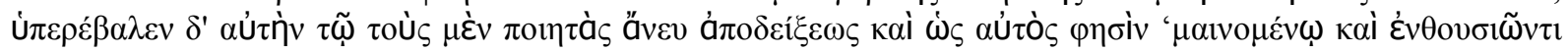

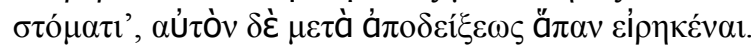

${ }^{30}$ Cfr. Herm. in Phdr. 75.1-79.3 e MOTTA 2013.

${ }^{31}$ Cfr. Hes. Th. 1-34.

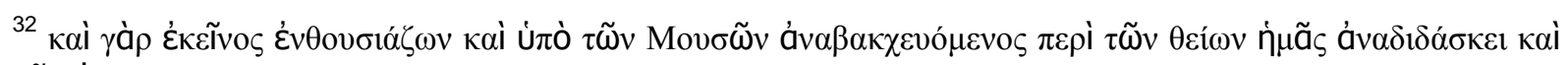

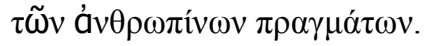

${ }^{33}$ Il punto di partenza dell'interpretazione filosofico-mistagogica dei miti si trova, secondo i Platonici, nel Fedro e nel concetto di mania. Attraverso l'analisi di questo concetto è possibile stabilire e dimostrare che Platone non nega (e anzi è ben consapevole di) una stretta relazione tra divinazione, misteri e poesia. Filosofia, divinazione, misteri e poesia sono strettamente connessi e rientrano nel percorso educativo che i giovani sono tenuti a seguire.

${ }^{34}$ Cfr. anche Procl. in R. II 193, 14-16.

${ }^{35}$ Cfr. PALUMBO 2011.

${ }^{36}$ Cfr. Procl. in R. II 159.3-4 e Anon. Proll. 8.1-17.

${ }^{37}$ Cfr. BUFFIÈRE 1956, p. 29; CARDULLO 1985: 101; LAMBERTON 1986: 170.
} 
interno $^{38}$, la forma piuttosto che la materia. Questa è la conclusione cui ci conducono i Prolegomeni, conclusione che sembra, però, escludere i diversi e ancora più alti gradi di verità presupposti dai testi dai quali il manuale alessandrino dipende.

\section{MATERIA E DECORO DEI MITI PLATONICI}

L'Anonimo dei Prolegomeni afferma la superiorità dell'espressione linguistica platonica rimarcando che i poeti comunicano in preda all'enthousiasmos, Platone, invece, attraverso dimostrazioni, ovvero attraverso logica e persuasione, l'unico modo per parlare sul serio $^{39}$, cioè - come scrive Proclo ${ }^{40}$ - l'unico modo per legare la verità con il vincolo dell'evidenza. Parlare seriamente implica utilizzare i miti con decoro ed è proprio in campo mitopoietico che il filosofo vince - secondo l'Anonimo - il confronto con Omero ${ }^{41}$. Il decoro $^{42}$ dei miti rende questi ultimi convenienti e cioè somiglianti a ciò di cui sono immagine: infatti, Platone non ha rappresentato in maniera oltraggiosa e blasfema gli dèi ${ }^{43}$. Mimetes massimamente mitetico ${ }^{44}$, nel costruire i suoi miti, il filosofo si è servito «in maniera mistica» - scrive Proclo - di immagini in grado di educare su questioni divine ${ }^{45}$. Tali immagini si sono trasformate in ritratti somiglianti agli dèi come lo sono le statue visibili ${ }^{46}$, affini a ciò che rappresentano per forma. Platone, quindi, riproduce - secondo i Neoplatonici - la divinità, attraverso le parole, con lo stesso decoro con cui le statue, fatte di marmo o di bronzo, la rappresentano. Il filosofo è, pertanto, scultore di agalmata e, analogamente al demiurgo divino, che plasma il sensibile animandolo come immagine dell'intelligibile ${ }^{47}$, è in grado di creare immagini e somiglianze che non stravolgono la natura degli dèi, ma

\footnotetext{
${ }^{38}$ Cfr. Procl. in R. 84.4-6.

${ }^{39}$ Cfr. Pl. Euthd. 288b.

${ }^{40}$ Cfr. Procl. in R. I 73.16-30; 158.16-159.6; 172.6-12

${ }^{41}$ Cfr. Anon. Proll. 7.24.

${ }^{42}$ Sul decoro dei miti cfr. Olymp. in Grg. 238.5-10.

${ }^{43}$ Cfr. Xenoph. DK21B 11. Su questo frammento cfr. PALUMBO 2004.

${ }^{44}$ Cfr. Procl. in Cra. XIV, 5.17.

${ }^{45}$ Cfr. Procl. in R. I 73, 16-22.

${ }^{46}$ Cfr. COULTER, 1976: 48 e MÜRI 1976: 31.

${ }^{47}$ Cfr. Anon. Proll. 13.15-29. Nel Timeo (37c) il mondo è agalma degli dèi eterni.
} 
conducono verso l'assimilazione al divino ${ }^{48}$. È Plotino il primo neoplatonico ad affermare che le statue degli dèi, frutto di un'arte superiore, partecipano del divino nel loro essere rappresentazione diretta delle realtà intelligibili: proprio per tal motivo esse consentono la mistica unione ${ }^{49}$. Queste immagini, ricettacoli di potenze divine, sono accessibili anche ai più, poiché - come spiega Porfirio prima di Proclo - sono sympoloi («congeneri») ai sensi umani e rappresentano l'invisibile attraverso modelli visibili ${ }^{50}$.

La dignità formale del mito è quindi salvaguardata da immagini riconosciute "legittime", perché esse sono in grado di rispettare quelle regole che corrispondono agli schemi teologici esposti da Platone in $R$. II $377 \mathrm{~d}-383 \mathrm{c}^{51}$ e commentati da Proclo ${ }^{52}$ e ancora prima da Sallustio ${ }^{53}$, allievo di Giamblico. L'Anonimo riprende questo aspetto della riflessione neoplatonica sul decoro quando afferma che il primo schema riguarda la bontà del dio, mentre il secondo si riferisce al fatto che la realtà divina è immutabile e veridica, cioè priva di inganno e falsità:

Dunque bisogna sapere che il Dio è buono e non produce mai falsità, né attraverso l'ignoranza della verità né attraverso alcun inganno; e che ogni Dio è immutabile e immobile, infatti non si trasforma né in ciò che è peggiore né in ciò che è migliore, perché, per quanto concerne il primo punto, non è nella sua natura e, quanto al secondo, non è possibile: è contrario alla sua natura trasformarsi in ciò che è peggiore, non può d'altra parte trasformarsi in ciò che è migliore, proprio perché è per essenza migliore di tutte le cose (Anon. Proll. 7.28-35, trad. MOTTA 2014) ${ }^{54}$.

È evidente che la filosofia si occupa anche degli dèi, e quindi è essa stessa teologia, o meglio una scienza teologica che indaga secondo princìpi e metodologie rigorose quelle cause trascendenti del mondo fisico che sono anche divinità ${ }^{55}$. I Neoplatonici definiscono gli aspetti di tale scienza ammettendo che le dottrine sugli dèi hanno origine in un passato mitico che

\footnotetext{
${ }^{48}$ Sulla homoiosis theo(i) cfr. supra n. 4.

${ }^{49}$ Cfr. Plot. IV 3 [27], 11.1-15 e IV 3 [27], 10.17-30. Cfr. FAZZO 1977, pp. 151-180.

${ }^{50}$ Cfr. Porph. Simul. fr.1.

${ }^{51}$ Cfr. FERRARI 1998; FERRARI 2006: 38-41; FERRARI 2014: 39-52.

${ }^{52}$ Cfr. Procl. in R. I 27.9-28.14 e 41.3-29.

${ }^{53}$ Cfr. Sallust. 4, 1.

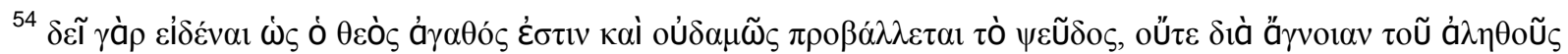

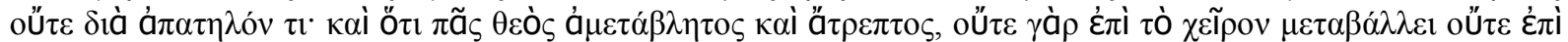
$\tau$ $\tau$

${ }^{55}$ Cfr. ancora FERRARI 1998.
} 
non è possibile rinnegare, poiché appartiene all'enciclopedia della paideia ellenica, e che pertanto è necessario presentare e spiegare adeguatamente:

Non bisogna intendere in modo così superficiale ciò che viene detto in forma di miti. E infatti sappiamo anche noi che una sola è la Causa Prima, il Dio, perché non vi sono molte cause prime (Olymp. in Grg. 32.15-17) ${ }^{56}$.

Il passaggio di Olimpiodoro sottolinea l'importanza di un'interpretazione "non letterale" del mito, interpretazione che viene esclusa nella Repubblica e sulla quale i Prolegomeni tacciono, perché tale testo viene letto nelle scuole preliminarmente a Platone e si rivolge quindi a un gruppo di aspiranti Platonici i quali non sono stati ancora iniziati ${ }^{57}$. Pertanto le loro facoltà raziocinante e intellettiva non si sono pienamente sviluppate: essi non sanno cosa sia il significato letterale e quello allegorico di un testo e quindi non sono in grado di cogliere il senso del mito omerico. Ecco uno dei motivi per cui nell'anonimo testo alessandrino non c'è alcun accenno all'importanza del mito arcaico per il completamento dell'anagoge, ma si discute solo di miti filosofici, ossia di quella categoria di miti che, fabbricati come agalmata nel rispetto degli schemi teologici, Proclo - come vedremo definisce paideutici. Se, infatti, in questa fase dell'apprendimento filosofico non si adottassero alcuni accorgimenti e non si selezionassero le letture si giungerebbe alla conseguenza prospettata da Platone:

Un giovane infatti non è in grado di giudicare ciò che è il senso nascosto e ciò che non lo è, ma ciò che ha accolto a questa età fra le sue opinioni suole diventare difficile da cancellare e immutabile (Pl. R. 2, 378d 7-e 2) ${ }^{58}$.

Il passo platonico, citato esplicitamente da Proclo e Olimpiodoro $^{59}$, mostra innanzitutto l'interesse filosofico e la finalità educativa dei commentatori. Quello verso la verità è un cammino graduale che guarda alla cura dell'anima, cura che può guarirla dal contatto con la materialità e così consentire il progresso verso il divino attraverso selezionati testi antichi. Gli unici miti cui si riferisce l'Anonimo sono quelli didattici, quelli cioè destinati

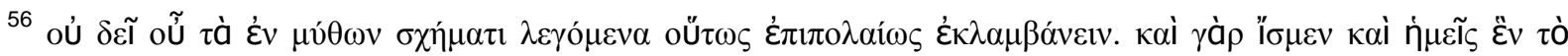

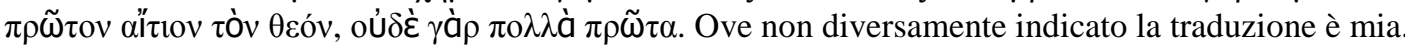

${ }^{57}$ Sul cursus studiorum cfr. MOTTA, 2014, pp. 21-25.

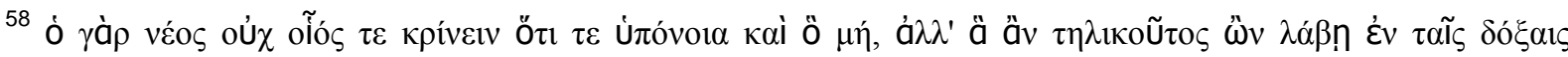

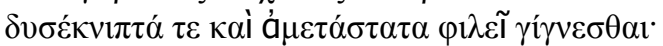

${ }^{59}$ Cfr. Procl. in R. I 79.29-80.4 e Olymp. in Grg. 239.3-11.
} 
a un'immediata comprensione: non ingannano con apparenze e non si concludono, come le favole di Esopo, con la spiegazione esplicita dell'insegnamento morale, insegnamento che l'aspirante filosofo può e deve imparare a cogliere da solo ${ }^{60}$. Il mito filosofico si serve di procedimenti scientifici, quali le dimostrazioni, per esprimere la verità ed è, seppur solo in un certo senso, simile all'epimython esopico che, benché falso, ha un messaggio intrinseco utile. È tuttavia superiore a tale racconto perché i miti filosofici fanno apparire immediatamente (eutheos) il fine della storia: «Del resto, il mito non è niente altro che un discorso falso che offre una rappresentazione di verità (Olymp. in Grg. 237, 18-19)» ${ }^{61}$. Falso per natura il racconto paideutico è ritenuto un mito che però è soprattutto un $\log _{0} s^{62}$ in quanto discorso e immagine verisimile ${ }^{63}$ che, riproducendo l'intelligibile nel sensibile, fa sì che il riconoscimento della somiglianza dia inizio al processo epistrofico di ritorno al paradigma. Il mito filosofico ha pertanto una falsità relativa, è una nobile menzogna ${ }^{64}$ con una propria utilità e una propria finalità di mediazione nei processi conoscitivi, una menzogna che, immagine imperfetta del modello perfetto ${ }^{65}$, trae la sua nobiltà dal riprodurre per somiglianza e dal molteplice verso l'Uno la via ontologica che invece procede per dissomiglianza dall'Uno al molteplice.

Se dunque il mito è immagine di verità e anche l'anima è immagine delle realtà prima di lei, è naturale che l'anima tragga piacere dai miti come immagine da immagine (Olymp. in Grg. 237.19-21) ${ }^{66}$.

Come si diceva, è all'anima, cioè alla facoltà psichica immaginativa, essa stessa un'immagine dell'Intelletto ${ }^{67}$, che si rivolge il mito, perché l'anima è in grado di risalire dagli aspetti molteplici del sensibile all'essenza degli enti ${ }^{68}$. Il mito paideutico di cui parlano i

\footnotetext{
${ }^{60}$ Cfr. Anon. Proll. 7.35-42.

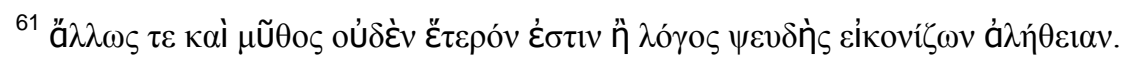

${ }^{62}$ Cfr. Pl. Grg. 523a 1-3. Cfr. Olymp. in Grg. 243.12-15 e 266.15-20.

${ }^{63}$ Sulla verosimiglianza delle immagini cfr. CERRI 1991: 19-28.

${ }^{64}$ Cfr. Pl. $R$. II 382c-d4. Sulla nobile menzogna cfr. CALABI 1998.

${ }^{65}$ Sulle tappe ascensive e sulla dialettica analogica cfr. BEIERWALTES 1990: 312-371.

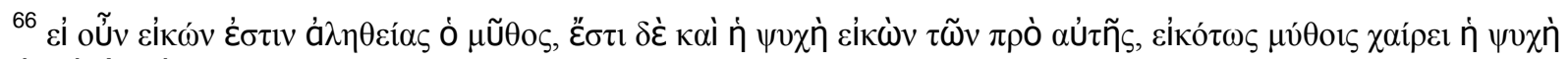

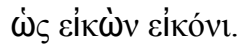

${ }^{67}$ Su ciò PÉPIN 1958: 176-177; El. in Porph. 27.15-16; ps. El. in Porph. 18.14; Dam. in Phd. II $\S 130$ (e il commento di GRITTI, 2012, pp. 59 ss.). Il motivo per cui traiamo piacere dai miti è esaminato da SHEPPARD, 1980, pp. 157-158.

${ }^{68}$ Cfr. Pl. Phd. 61b.
} 
Neoplatonici non è mettere in musica le favole di Esopo, ma comporre logoi filosofici che rappresentino la verità per far nascere nell'anima dei giovani lettori un piacere cognitivo ${ }^{69}$, piacere che consente di superare il piano immediatamente immaginativo e raggiungere quello epistemologico.

\section{MATERIA E OSCENITÀ DEI MITI OMERICI}

Solo pochi aspiranti filosofi diventeranno Platonici e solo questi sapranno in che modo anche il mito omerico, benché non sembri rispettare quel decoro su cui richiama l'attenzione l'Anonimo, è rivelatore di verità, cioè come i poeti hanno insegnato a Platone l'ordine degli esseri.

Ed essi [scil. i miti] sono nati anche in modo che le dottrine non vengano tramandate a caso; infatti come nei templi gli strumenti sacri e i riti misterici hanno drappi, affinché gli indegni non li contemplino a caso, così anche in questo ambito i miti sono veli delle dottrine affinché non siano nude e accessibili a chiunque voglia (Olymp. in Grg. 239.12-17) ${ }^{70}$.

I miti cui si riferisce Olimpiodoro sono quelli che custodiscono verità essenziali e che, attraverso l'uso di veli - simili ai drappi che nei riti misterici coprono gli strumenti sacri -, si difendono da profanatori e profanazioni. L'educazione filosofico-poetica si conclude con miti che hanno analogie con la pratica più esoterica della tarda antichità, pratica che nel cursus filosofico corrisponde alla teurgia ${ }^{71}$. Quest'ultima viene introdotta nel neoplatonismo successivo a Plotino per superare i problemi connessi alla mancata accettazione, da parte dei Neoplatonici tardi, della teoria plotiniana dell'anima non-discesa, teoria propria della cosiddetta corrente razionalista. A tale teoria si oppone quella della corrente teurgica secondo la quale, poiché l'anima discende senza residui, è necessario ricreare un contatto col divino attraverso alcune pratiche magiche ${ }^{72}$. Per Plotino, sebbene il vivere fra le cose terrene sia

69 Cfr. Anon. Proll. 15.24 e Arist. Po. 1448b 4-9. Sulla mímēsis, Platone e la Poetica di Aristotele cfr. PALUMBO 2008: 488-543.

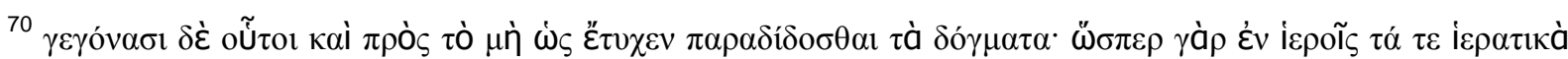

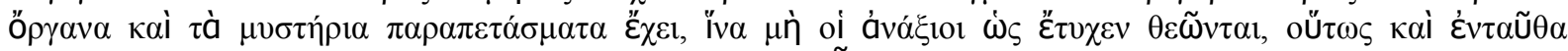

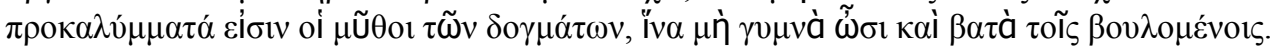

${ }^{71}$ Sulla teurgia cfr. VAN LIEFFERINGE 1999.

${ }^{72}$ La contrapposizione tra corrente razionalista e corrente teurgica è tuttavia - come dimostrano recenti studi troppo schematica; cfr., per es., GRITTI 2008: 67-89. Sui presupposti psicologici dell'attività teurgica cfr. SHAW 1995. 
sempre caduta, esilio e perdita delle ali, una parte dell'anima continua a essere nell'intelligibile ${ }^{73}$. Invece, in numerosi passi procliani ${ }^{74}$ si legge - come aveva affermato, per primo, Giamblico - che ogni anima particolare, quando scende nel mondo del divenire, scende interamente ${ }^{75}$ : infatti, sarebbe assurdo pensare che la più alta parte dell'anima, rimanendo sempre perfetta, non possa controllare le altre facoltà e rendere anche queste perfette. Per Giamblico il processo filosofico di ritorno all'Uno è compromesso dal forte legame dell'anima al corpo, anima che ha perso contatto con gli intelligibili e quindi non riesce a riconoscersi in perenne possesso del vero. In questa fase nel neoplatonismo per conoscere e raggiungere il divino non è più possibile la sola contemplazione noetica, ma sono necessari dei riti, riti teurgici, il cui successo è determinato solo dagli dèi. L'importanza di tali pratiche è riconosciuta dai Neoplatonici secondo i quali Platone stesso già le indica come mezzi di risalita all'intelligibile.

Figure legate a tali pratiche sono i teurgi, detentori di un sapere rituale superiore alla pura intellezione, sapere che è estraneo alle masse, e che appare quindi una mystagogia collegata direttamente alla rivelazione contenuta negli Oracoli Caldaici. Scrive l'imperatore Flavio Claudio Giuliano, la cui classificazione dei mythoi è molto vicina a quella procliana e probabilmente (e non a caso, direi) debitrice di una fonte comune quale poteva essere stata Giamblico di cui l'Apostata era fedele discepolo:

«La natura ama nascondersi» e non sopporta che il segreto dell'essenza degli dèi venga trasmesso a orecchie impure con parole nude. Per questo è utile la natura ineffabile e sconosciuta dei caratteri: essa cura non soltanto le anime, ma anche i corpi, e provoca le apparizioni divine (Iul. or. 7, 216c 1-7; trad. DE VITA 2011) ${ }^{76}$.

Come nei Prolegomeni è un frammento dell'oscuro Eraclito - filosofo che con Senofane è annoverato tra gli iniziatori dell'antica discordia tra poesia e filosofia ${ }^{77}-$ a sottolineare il carattere di quel mito proprio dei poeti ispirati e che riguarda l'iniziazione misterica alla teologia, così nel passaggio giulianeo è un frammento eracliteo a introdurre la

\footnotetext{
${ }^{73}$ Plot., Enn. IV, 8 [6], 8.11-13.

${ }^{74}$ Cfr. BLUMENTHAL 1997: 269-290.

${ }^{75}$ Procl. Inst. 211.

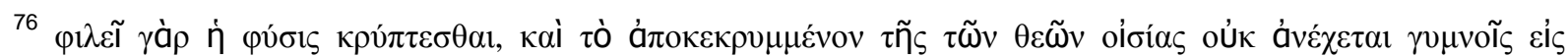

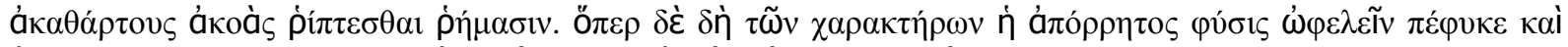

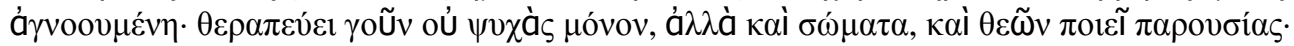

${ }^{77}$ Cfr. Heracl. DK22B 40, 42, 56, 57, 106 DK; Xenoph. DK21B 1, 11, 12, 14, 15, 16; Pl. R. 10, 607b 5-6. Cfr. KANNICHT, 1988.
} 
necessità di nascondere la vera natura degli esseri agli impuri. Nel testo giulianeo viene espresso, con allusioni terminologiche alle cerimonie teurgiche caldaiche e attingendo al lessico dei misteri, lo scopo cui questi riti mirano: essi consentono di curare non solo l'anima ma anche il corpo nel quale l'anima è completamente immersa. Nel tardo neoplatonismo la psicologia influenza diversi aspetti della filosofia tra cui appunto quello epistemologico. La condizione in cui l'anima si trova influenza la sua capacità di conoscere e comporta un processo epistrofico più complesso in cui l'iniziazione va intesa in senso non solo strettamente filosofico ma anche religioso: tale iniziazione è quella che - come scrive Sallustio nel già citato capitolo quarto del suo De diis quando affronta la questione dell'uso dei miti e del perché siano divini - consente la comunicazione con gli dèi.

Le storie mitiche dei poeti contengono un potere iniziatico, e quindi mistagogico, il quale sconcerta l'anima $^{78}$ obbligandola non ad attivare il processo immaginativo che costruisce la conoscenza per somiglianza, ma a cercarne il senso nascosto, a cercare dietro i drappi dell'oscenità del mito la verità ${ }^{79}$. Ecco il motivo per il quale i miti usati da Omero non possono essere compresi se non vengono considerati come delle provocazioni che stimolano l'anima dell'iniziato alla ricerca. Nel Commento alla Repubblica, Proclo, dopo aver discusso l'esoterismo degli insegnamenti orfici e dopo aver elaborato la sua teoria del simbolo, scrive che la mitologia ha due finalità separate, una per la corretta educazione dei giovani - che è quella su cui si concentra, a nostro giudizio, l'Anonimo riprendendo i contenuti e il linguaggio usati dal diadoco ateniese -, l'altra per la ieratica e la simbolica evocazione del divino $^{80}$. Qui e in altri luoghi del Commento alla Repubblica e nella Teologia Platonica viene discussa la differenza tra i miti platonici e quelli omerici: gli uni sono paideutikoi e philosophoteroi, perché rivelano verità filosofiche ${ }^{81}$, mentre gli altri entheastikoteroi solo a pochi consentono di completare il cammino di conoscenza con l'unione mistica ${ }^{82}$, fine ultimo di ogni Platonico. Convinto del forte potere che la teurgia svolge in tale contesto ${ }^{83}$, Proclo

\footnotetext{
${ }^{78}$ Cfr. Procl. in R. I 84.22-29; Olymp. in Grg. 41, 2.14-15 e Anon. Proll. 7. 25-35. Vd. TROUILLARD, 1977, p. 36. Tale sconcerto prende gli uomini ben disposti: la predisposizione è, infatti, un prerequisito che maestri Medio e Neoplatonici valutano prima dell'insegnamento: cfr. Alb. Intr. V 148.21-149.31; Amm. in Cat. 6.22-23; Marin. 22; Olymp. Proll. 10.3-5; 10.10; Anon. Proll. 15.60-65.

${ }^{79}$ Cfr. Procl. in R. I 85.26-86.5 e Theol. Plat. VI 23, 103.29-104.3.

${ }^{80}$ Procl. in R. I 84.25-26.

${ }^{81}$ Cfr. Procl. in R. I 79.5-13.

${ }^{82}$ Procl. in $R$. I 81.10.

${ }^{83}$ Giamblico (Myst. 96.11-97.1) dice che la filosofia speculativa non conduce all'unione con gli dèi.
} 
ritiene che la superiorità dei miti ispirati sia dovuta al fatto che essi concordano «con tutte le pratiche ieratiche, con le iniziazioni, con i misteri e con le epifanie degli dèi» ${ }^{84}$.

L'eccellenza di questi miti non è nell'essere educativi, ma nell'essere mistificativi, poiché non sono rivolti ai giovani, ma alle persone più mature. Anche Socrate lo dimostra quando dice che questi miti sono adatti a essere ascoltati solo da un gruppo scelto in segreto, che compie un sacrificio non di un maiale ordinario, ma di un'enorme e non procurabile vittima. Lontano dal disprezzare, come molti credono, questo metodo mitopoietico, si fa ascoltatore di miti paralleli alle più sante iniziazioni e ai più perfetti misteri (Procl. in $R$ I 80.11-20) ${ }^{85}$.

Proclo, quindi, afferma - richiamandosi direttamente all'auctoritas di Socrate e non alle interpretazioni dei molti - la superiorità del mito omerico, mito creato da un poeta ispirato direttamente dalla divinità, il quale vela il divino attraverso ciò che appartiene all'umano e, richiamando la trascendenza attraverso ciò che è immanente, non crea rassomiglianze, bensì priva di ogni verosimiglianza la sua narrazione. Come scrive l'imperatore Giuliano, è proprio l'inverosimiglianza a essere utile in questi miti, perché essa funge da stimolo al raggiungimento di un senso nascosto ${ }^{86}$. Infatti, benché inverosimile (e anzi proprio perché inverosimile), l'antropomorfizzazione omerica è l'unico modo per comunicare il divino, cioè la trascendente superiorità degli dèi: evidenziare la distanza, la dissomiglianza e non la somiglianza, tra umano e divino contribuisce, in questo caso e a questo grado di iniziazione, a suscitare il desiderio di saggezza che è nascosto nei miti sugli dèi ${ }^{87}$. Pertanto non è possibile affermare che Omero rappresenta gli dèi, perché egli non prende a modello il divino, ma - in taluni casi - lo nasconde, e così facendo non è mimetico come Platone, non crea immagini rassomiglianti, ma dissomiglianti come lo sono i simboli e i segni. Allora, proprio poiché si serve di simboli e segni, Omero sfugge alla critica costruita contro la mimesis poetica nella Repubblica ${ }^{88}$ e la sua poesia ispirata si pone a un livello superiore rispetto a quella mimetica che, nella gerarchia tripartita delle varietà di vita, Proclo associa alla forma più bassa. Pur essendo per Proclo la poesia tutta mimetica sembra, a questo

\footnotetext{
${ }^{84}$ Procl. in R. I 110.23-25.

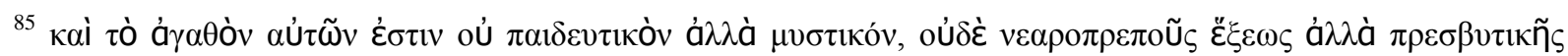

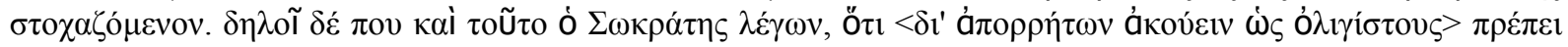

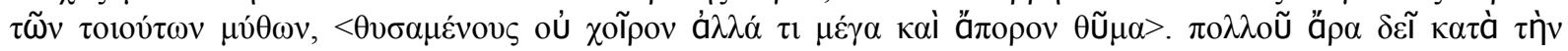

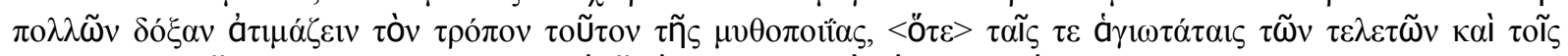

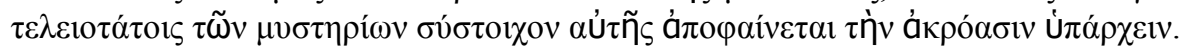

${ }^{86}$ Iul. or. 7, 219a.

${ }^{87}$ Cfr. Procl. in R. I 85.26-86.1.

${ }^{88}$ Cfr. Pl. R. 2, 377e-383c. Su Proclo e Omero cfr. DOMARADZKI 2014.
} 
punto, esistere una forma di poesia senza mimesis ${ }^{89}$. Essa va riconosciuta in quella che si serve di oggetti privi di affinità o somiglianza (anomoios): del resto, il diadoco ateniese afferma esplicitamente che un poeta ispirato, il quale rivela la verità sugli esseri attraverso segni, non è un mimetes $^{90}$.

La mimesis che fallisce la somiglianza (anomoios: Pl. $R .2,388 \mathrm{c} 3$ ) e che conduce a una rappresentazione indegna (anaxios: Pl. $R$. 388d 3) non viene tuttavia condannata da Proclo. Anzi la rivelazione di un poeta ispirato direttamente dalla divinità avviene, come il fenomeno della divinazione, «senza dire e senza nascondere, ma accennando» - scrive Giamblico $^{91}$, citando Eraclito $^{92}$-: essa quindi non si rivolge alla facolta psichica immaginativa che procede attraverso il riconoscimento della somiglianza delle immagini, ma, utilizzando simboli, stimola la nostra mente a raggiungere, direttamente per il tramite della divinità, l'unione mistica. È attraverso la costruzione sapiente dell'analogia tra teurgia e miti omerici, idea nata probabilmente nella speculazione giamblichea - come potrebbero suggerire le riflessioni di Salustio e Giuliano -, che Siriano e il grande diadoco ateniese riabilitano Omero e lo presentano come un teologo ${ }^{93}$.

Per stabilire una relazione con il divino, la teurgia si serve di strumenti i quali si relazionano ai loro referenti attraverso un rapporto simpatetico ${ }^{94}$. Allo stesso modo il mito omerico riveste l'essenza trascendente e inconoscibile degli dèi con segni e simboli ${ }^{95}$ che poi offre ai Platonici, disposti a lasciarsi guidare da queste tracce e desiderosi di trovare, dopo essersi purificati, la mistica unione con la divinità nelle sacre iniziazioni ${ }^{96}$. Proclo non avvicina quindi solo Omero a Platone, ma assimila tutta la filosofia, in cui rientra anche la rappresentazione oscena del divino, ossia la dissomiglianza dell'umano e del divino, a una

\footnotetext{
${ }^{89}$ Sulle contraddizioni nel discorso procliano e sulla difficoltà del diadoco ateniese di distinguere chiaramente mimesi e simbolismo cfr. HALLIWELL 2009: 267-284.

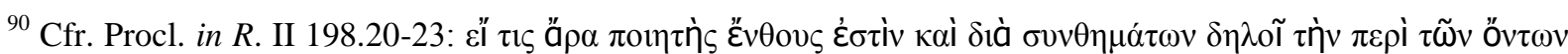

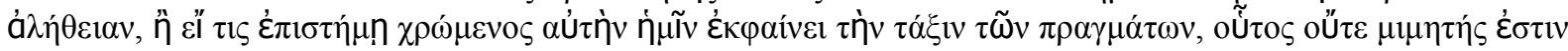

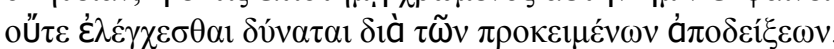

${ }^{91}$ Iambl. Myst. 136.2-10.

${ }^{92}$ Cfr. Heracl. DK22B 93.

${ }^{93}$ Cfr. COULTER 1976: 50-57;

${ }^{94}$ Cfr. Procl. Inst. 145. Cfr. BARBANTI 1983: 98-113; 131-140 e 171. Il passaggio tratto direttamente da R. 378 a 4-5 prova che la poesia omerica può essere considerata una mistagogia (Procl. in R. I 80.20-23).

${ }^{95}$ Il diadoco afferma esplicitamente comparando l'impatto dei simboli nei miti con quello dei simboli nei riti magici (in $R$. I 78.18-79.4).

${ }^{96}$ Cfr. Procl. in R. I 78.17-79.4.
} 
mistagogia ${ }^{97}$ che, prima con il linguaggio della scienza e poi con quello misterico della teurgia, guida l'anima all'epopteia ${ }^{98}$.

\section{ALCUNE OSSERVAZIONI CONCLUSIVE}

Ben consapevole che non è questa la sede adeguata per tentare di risolvere la questione della relazione tra mito filosofico e mito poetico, vorrei comunque segnalare alcune suggestioni. Scrive Aloys de Marignac che il mito è una forma di verità (DE MARIGNAC 1951: 28) anche se le immagini costruite dai poeti - sottolinea Pierre Louis - si distinguono da quelle di Platone che «ne sont pas des simples ornement (LOUIS 1945: 6)». Quest'ultima riflessione - come si è cercato di discutere - non è sempre condivisibile e certamente non è applicabile alla filosofia dei Neoplatonici di Atene e di Alessandria, perché la religione omerica non è considerata inferiore in quanto espressione di un'età primitiva, anzi il mito, di cui essa si serve, esprime una delle forme più alte di verità rivelata, verità cui, per giunta, hanno accesso pochi iniziati. A un certo punto della storia del platonismo la teologia diventa il culmine della riflessione filosofica: il suo linguaggio "purifica" il mito omerico e lo riconfigura in un sistema filosofico che non è nuovo, poiché dichiara di seguire, e di fatto segue, l'intera tradizione filosofico-religiosa greca. All'interno di essa Platone, autore e poeta di dialoghi, "compete” con Omero, il quale, triomphateur (BUFFIÈRE 1956: 29) dopo l'esilio dalla kallipolis, sembra per certi aspetti superare in divinità anche il divino filosofo che, invece, appare "soltanto" il massimo mimetes. In realtà la discussione neoplatonica non rende Platone inferiore a Omero: il filosofo, theios aner, sa usare il potere mimetico delle immagini, sa rivelare il divino attraverso un linguaggio adeguato, laddove Omero, mancando del linguaggio della scienza - secondo l'Anonimo -, non è capace di spiegare ciò che dice in preda all'enthousiasmos ${ }^{99}$. Platone viene, quindi, difeso, da Proclo e dall'Anonimo, dall'accusa di essere un poeta e non uno scienziato $^{100}$ : la riflessione sul linguaggio della scienza e quella sul linguaggio del simbolo sono certamente alcune delle chiavi di lettura attraverso le quali è possibile comprendere come i Neoplatonici riabilitino Omero e al

\footnotetext{
${ }^{97}$ Questo tipo di poesia è proprio «una mistagogia e una iniziazione che eleva le anime degli ascoltatori (Procl. in $R$. I 80.22-23)».

${ }^{98}$ Cfr. BRISSON 1996: 122.

${ }^{99} \mathrm{Su}$ questa debolezza della poesia omerica cfr. Pl. Ap. 22a-e.

100 Contra CERRI 1991: 53-55, il quale ritiene che Proclo difenda Platone con argomentazioni piuttosto astratte e involute. Cfr. Procl. in R. I 105.23-26.
} 
contempo attribuiscano alla mimesis platonica caratteri di scientificità indiscutibilmente $\operatorname{divina}^{101}$.

La teurgia resta comunque un argomento "scomodo" per l'Anonimo dei Prolegomeni che, pur sottolineando l'importanza di Omero per la formazione di Platone e benché debitore della lezione giamblichea e procliana ${ }^{102}$, a questo punto dell'istruzione scolastica, e cioè all'inizio dei «grandi misteri», non ritiene opportuno neppure accennare al potere dei miti poetici $^{103}$. A differenza dell'Anonimo, per Proclo e Olimpiodoro il mito poetico è una forma espressiva che rientra a pieno titolo nella neonata scienza filosofica.

\section{RIFERIMENTI BIBLIOGRAFICI}

ABBATE, Michele (2005). Proclo, 'Teologia Platonica', Bompiani, Milano.

BARBANTI, Maria (1983). Proclo tra filosofia e teurgia, Bonanno, Catania.

BEIERWALTES, Werner (1990). Proclo. I fondamenti della sua metafisica, trad. it., Vita e Pensiero, Milano.

BLUMENTHAL, Henry J. (1997). “The Psychology of Plotinus and Later Platonism”, in J. J. Cleary (ed.), The Perennial Tradition of Neoplatonism, University Press, Leuven.

BOUFFARTIGUE, Jean (1987). "Représentations et évaluations du texte poétique dans le Commentaire sur la République de Proclos", in M. Costantini et. al., Le texte et ses représentations, Presses de l’Ecole Normale Supérieure, Paris, pp.129-143.

BRISSON, Luc (1996). Introduction à la Philosophie du mythe, 1. Sauver les Mythes, Vrin, Paris 1996.

BRISSON, Luc (2000). "Nascita di un mito filosofico: Giamblico (VP 146) su Aglaophamos", in M. Tortorelli Ghidini, A. Storchi Marino, A, Visconti, Tra Orfeo e Pitagora. Origini e incontri di culture nell'antichità, Bibliopolis, Napoli, pp. 237253.

\footnotetext{
${ }^{101}$ Sulla mimesis platonica che non può essere bandita cfr. Procl. in R. II 158.26-159.6. e in R. II 162.27-163.9.

${ }^{102}$ Cfr. MOTTA, 2014, pp. 26-30.

${ }^{103}$ Cfr. MOTTA, 2014, pp. 30-34; 51-53; 72-76.
} 
BRISSON, Luc (2004). How Philosophers Saved Myths: Allegorical Interpretation and Classical Mythology, tr. by C. Tihanyi, The University of Chicago Press, ChicagoLondon.

BUFFIÈRE, Félix (1956). Les mythes d'Homère et la pensée grecque, Les Belles Lettres, Paris.

BUXTON, Richard (1999). From Myth to Reason?, Oxford University Press, Oxford.

CALABI, Francesca (1998). "La nobile menzogna", in M. Vegetti, Platone, La Repubblica, vol. II, Bibliopolis, Napoli, pp. 445-457.

CALAME, Claude (1999). Mito e storia nell'Antichità greca, Dedalo, Bari.

CARDULlO, Loredana (1985). Il linguaggio del simbolo in Proclo, Università di Catania, Catania.

CENTRONE, Bruno (1991). Platone, 'Fedone', Laterza, Roma-Bari.

CERRI, Giovanni (1991). Platone sociologo della comunicazione, Il Saggiatore, Milano.

COLLI, Giorgio (1975). La nascita della filosofia, Adelphi, Milano.

CORNFORD, Francis (1952). Principium sapientiae: the origins of Greek philosophical thought, University Press, Cambridge.

COULTER, James (1976). The Literary Microcosm. Theories of Interpretation of the Later Neoplatonists, Brill, Leiden.

DE MARIGNAC, Aloys (1951). Imagination et dialectique: essai sur l'expression du spirituel par l'image dans les dialogues de Platon, Les Belles Lettres, Paris.

DE VITA, Maria Carmen (2011). Giuliano filosofo imperatore neoplatonico, Vita e Pensiero, Milano.

DETIENNE, Marcel (1983). L'invenzione della mitologia, Bollati Boringhieri, Torino.

DOMARADZKI, Mikolaj (2014). "Symbolic Poetry, Inspired Myths and Salvific Function of Allegoresis in Proclus' Commentary on the Republic", Peitho/Examina Antiqua 1/5, pp. 119-137.

DÖRRIE, Heinrich - BALTES, Matthias - PIETSCH, Christian (2008). Die philosophische Lehre des Platonismus. Theologia Platonica I, Frommann-Holzboog, Stuttgart-Bad Cannstatt 2008. 
FAZZO, Vittorio (1977). La giustificazione delle immagini religiose dalla tarda antichità al cristianesimo. I. La tarda antichità, Edizioni scientifiche italiane, Napoli.

FERRARI, Franco (1998). "Theologia", in M. Vegetti, Platone, La Repubblica, vol. II, Bibliopolis, Napoli, pp. 403-425.

FERRARI, Franco (2006). I miti di Platone, Mondolibri, Milano.

FERRARI, Franco (2014). "Tra menzogna e verità: lo spazio del mito nella filosofia di Platone", in S. Rotondaro, Mythos e logos, Valentina Porfidio Editore, Moliterno, pp. 39-52.

GIANGIULO, Maurizio (1991). Giamblico, La Vita Pitagorica, BUR, Milano.

GRITTI, Elena (2008). Proclo. Dialettica anima esegesi, LED, Milano.

GRITTI, Elena (2012). Il vero nel mito. Teoria esegetica nel commento di Olimpiodoro Alessandrino al 'Gorgia', Aracne, Roma.

GUIDELLI, Chiara et alii (1997). Enneadi di Plotino, 2 voll., UTET, Torino.

HADOT, Pierre (1987). Esercizi spirituali e filosofia antica, (1987), tr., Einaudi, Torino.

HALLIWELL, Stephen (2009). L'estetica della mimesis: testi antichi e problemi moderni, tr. it., Aesthetica Edizioni, Palermo.

KANNICHT, Richard (1988). The ancient quarrel between philosophy and poetry: aspect of the Greek conception of literature, University of Canterbury, Christchurch.

KUISMA, Oiva (1996). Proclus' Defence of Homer, Societas Scientiarum Fennica, Helsinki.

LAMBERTON, Robert (1986). Homer the Theologian: Neoplatonist Allegorical Reading and the Growth of the Epic Tradition, University of California Press, Berkeley.

LAMBERTON, Robert (1992). The Neoplatonists and the Spiritualization of Homer, in R. Lamberton, J.J. Keaney, Homer's Ancient Readers. The Hermeneutics of Greek epic's earliest exegetes, Princeton University Press, Princeton - New Jersey, pp. 115133.

LAVECCHIA, Salvatore (2006). Una via che conduce al divino: la homoiosis theo nella filosofia di Platone, Vita e Pensiero, Milano.

LO SCHIAVO, Aldo (1983) = Omero filosofo: l'enciclopedia omerica e le origini del razionalismo greco, Le Monnier, Firenze.

LOUIS, Pierre (1945). Le métaphores de Platon, Les Belles Lettres, Paris. 
MANOLEA, Christina (2004). The Homeric Tradition in Syrianus, University of London, Thessaloniki.

MASULLO, Rita (1985). Marino di Neapoli, Vita di Proclo, M. D’Auria, Napoli.

MORESCHINI, Claudio (1996). “Motivi esegetici e filosofici negli Scholia in 'Phaedrum' di Ermia Alessandrino", Cassiodorus 2, pp. 99-117.

MOTTA, Anna (2013). "Immagini di Bellezza. Questioni di metafisica neoplatonica", Филозофија/Filozofija - A Journal of Philosophical Inquiry 35/2, pp. 27-42.

MOTTA, Anna (2014). Anonimo, Prolegomeni alla filosofia di Platone, Armando Editore, Roma.

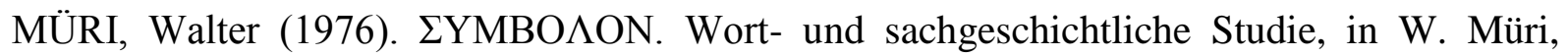
Griechische Studien. Ausgewählte wort- und sachgeschichtliche Forschungen zur Antike, F. Reinhardt, Basel, pp. 1-44.

PALUMBO, Lidia (2004). "Il frammento 1 D.K. di Senofane (= 1 Gent.-Pr.): appunti per una definizione della nozione di atmosfera simposiale", Atti dell'Accademia di Scienze Morali e Politiche 114, pp. 137-148.

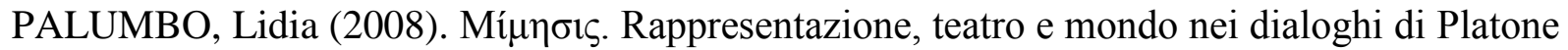
e nella Poetica di Aristotele, Loffredo, Napoli.

PALUMBO, Lidia (2011). "Mimesis ed enthousiasmos in Platone. Appunti sul Fedro", in G. Casertano, Il Fedro di Platone. Struttura e problematiche, Loffredo, Napoli, pp. 157172.

PÉPIN, Jean (1958). Mythe et Allégorie. Les origines grecques et les contestations judéochrétiennes, Aubier, Paris 1958.

PICHLER, Reinhard (2006). Allegorese und Ethik bei Proklos: Untersuchungen zum Kommentar zu Platons Politeia, Frank\&Timme, Berlin.

RAMELLI, Ilaria - LUCCHETTA, Claudio (2004). Allegoria, vol. 1: L'età classica, Vita e Pensiero, Milano.

RISPOLI, Gioia (1988). Lo spazio del verisimile. Il racconto, la storia e il mito, M. D’Auria, Napoli.

SAFFREY, Henri Dominique (1981). "Les néoplatoniciens et les oracles chaldaïques”, Revue Etudes Augustiniennes 27, pp. 209-225. 
SAFFREY, Henri Dominique (1992). Accorder entre elles les traditions théologiques : une caractéristique du néoplatonisme athénien, in E.P. Bos - P.A. Meijer, On Proclus and his Influence in Medieval Philosophy, Brill, Leiden - New York - Köln 1992, pp. 3550.

SHAW, Gregory (1995). Theurgy and the Soul, The Pennsylvania State University Press, University Park.

SHEPPARD, Anne (1980). Studies on the 5th and 6th Essays of Proclus' Commentary on the 'Republic', Vandenhoeck \& Ruprecht, Göttingen.

TROUILLARD, Jean (1977). "Les fondaments du mythe selon Proclos", in AA. VV. Le mythe et le symbole de la connaissance figurative de Dieu, Editions Beauchesne, Paris.

TROUILLARD, Jean (1982). La mystagogie de Proclos, Les Belles Lettres, Paris.

VAN DEN BERG, Robbert (2001). Proclus’ Hymns, Brill, Leiden - Boston - Köln.

VAN LIEFFERINGE, Carine (1999). La Theurgie : des Oracle chaldaïque à Proclus, Centre international d'Etude de la religion grecque antique, Liège.

VERNANT, Jean-Pierre (2007). Oeuvres : religions, rationalités, politique, Seuil, Paris. 